\title{
Use of QTL Analysis in Physiological Research ${ }^{1}$
}

\author{
D. Vreugdenhil ${ }^{a}$, M. Koornneef ${ }^{b, d}$, and L. I. Sergeeva ${ }^{a, c}$ \\ ${ }^{a}$ Laboratory of Plant Physiology, Wageningen University, Arboretumlaan 4, 6703 BD Wageningen, The Netherlands; \\ fax:31-317-4847; e-mail: dick.vreugdenhil@wur.nl \\ ${ }^{b}$ Laboratory of Genetics, Wageningen University, The Netherlands \\ ${ }^{c}$ Institute of Plant Physiology, Russian Academy of Sciences, Botanicheskaya ul. 35, Moscow, 127276 Russia; \\ ${ }^{d}$ Max Planck Institute for Plant Breeding Research, Carl von Linné Weg 10, Cologne, 50829 Germany
}

Received June 21, 2006

\begin{abstract}
Quantitative trait locus (QTL) analysis is a powerful approach to map and subsequently identify genes involved in complex traits. Here we describe the basic principles and recent achievements of this method, and its application in physiological research in plants. The rapidly increasing amount of molecular and "omics" data and genetic resources and tools, in model species (Arabidopsis) and crops, will greatly support and stimulate the use of this approach in the near future.
\end{abstract}

DOI: $10.1134 / \mathrm{S} 1021443707010025$

Key words: Arabidopsis - QTL - gene cloning - gene function - natural variation

\section{INTRODUCTION}

During the past decades, enormous progress has been made in the unraveling of gene functions in plants. This progress was partly based on the identification of genes causing a particular mutant phenotype. In other cases, descriptions of gene functions were based on analysis of the phenotype of mutants, in which the function of one particular gene had been disrupted without any bias to a specific phenotype. These approaches have been nicknamed forward genetics (from phenotype to gene) and reverse genetics (from gene to phenotype), respectively. Traits best amenable to one of these approaches usually show a discrete variation, and the difference between the mutant and wild type is limited to one gene (or more genes when mutations are combined by crossing).

However, in many cases, the difference in trait values between genotypes shows continuous variation, e.g. plant growth rate. This indicates that they are likely to be controlled by a number of genes, and/or are subjected to gene $\times$ environment interactions. The unraveling of the genes controlling such polygenic traits has only just begun. Here we will describe the usefulness and limitations of quantitative trait locus (QTL) analysis in this endeavor. Examples given will be mostly

\footnotetext{
${ }^{1}$ The text was submitted by the authors in English.

Abbreviations: HIF-heterogeneous inbred family; LOD—logarithm of odds; NIL—near isogenic family; QTL - quantitative trait locus; QTN - quantitative trait nucleotide; RIL-recombinant inbred lines; RT-PCR-reverse transcription polymerase chain reaction.
}

from recent research using the model plant Arabidopsis thaliana.

\section{WHAT IS QTL ANALYSIS?}

QTL analysis is based on the natural allelic variation present within a species. This allelic variation gives rise to smaller or larger quantitative effects on the trait under investigation. Natural allelic variation differs from induced variation (mutants), since the alleles occur in natural populations and are supposed to give selective advantage in certain conditions, or at least to be neutral. For QTL mapping a segregating population is required, derived from two parents. Preferably-but not necessarily - the parents of such a population should be divergent for the trait of interest. Since parental lines are taken from naturally occurring populations (named accessions or ecotypes for wild species, and cultivars, breeding lines, or landraces in crop species), they are likely to differ for many loci. QTL analysis implies the discovery of the association between trait values and the allelic status of the loci of all individuals in the segregating population. The genotype of individuals (or lines) can nowadays relatively easily be determined using molecular markers. QTL analysis reveals the genetic region(s) where a gene (or a set of closely linked genes) is located, which have a significant quantitative effect on the trait, and it describes the magnitude of the effect. This implies that a QTL is not a gene, but only a region on a chromosome, and hence it will require further effort to identify the gene(s) responsible for the variation in trait values within the QTL region.

For QTL mapping, it is very effective to use immortal mapping populations. Such populations have to be 


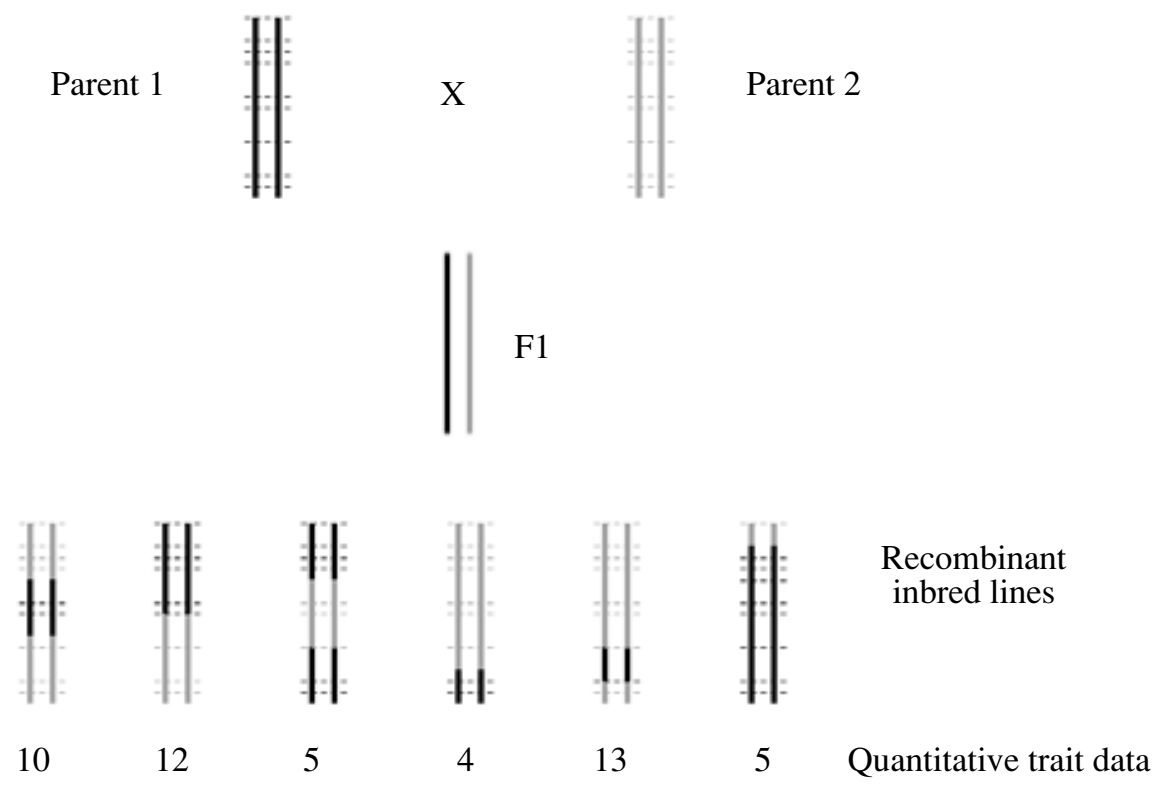

Fig. 1. Scheme showing the production of a set of recombinant inbred lines (RILs) and their use for QTL analysis. For the sake of simplicity only one chromosome per line is shown and only six RILs are represented.

Two parental lines (accessions), indicated with black and grey chromosomes, respectively, are crossed. The resulting F1 is selfed to generate F2 lines. RIL are generated by successive selfing of the F2 lines until homozygosity is achieved at the F8 generation or higher. Black and grey horizontal dashed lines indicate positions and genotype of molecular markers.

In this example, the quantitative trait data as given at the bottom of the figure, indicate a QTL at the lower end of the chromosome; the presence of alleles of parent 2 at the lowest marker is associated with a high value of the trait, whereas alleles from the other parent result in a low trait value (from [3], with permission).

genotyped only once, and can then be used for replicate experiments, on the same or different traits, or be used to analyze a particular trait under various environmental conditions, revealing possible genotype $\times$ environment interactions. Recombinant inbred lines (RILs) and backcross inbred or introgression lines are most frequently used. In Arabidopsis, RILs populations are obtained by repeated self-pollination and single seed descent of F2 obtained after crossing two divergent accessions. In this species, an increasing collection of RIL populations is becoming available and has been used to analyze a wide range of traits $[1,2]$. The principle of QTL mapping using a RIL population is schematically given in Fig. 1 [3].

The accuracy, with which a trait can be mapped, depends on three parameters: the density of the markers, the size of the population, and the accuracy by which one can determine the trait value (dealing with physiological and measurement variation), which can be described as the heritability (which part of the variation observed is due to genetic factors). The larger the population, the larger the number of recombination events, thus increasing the accuracy of mapping. The number of markers has to be balanced with the size of the population, or rather the number of recombinations. Indefinitely increasing marker density does not help much, since it will not result in a larger number of recombination events. However, sufficient markers should be put on the map to accurately reveal the recombination sites. In Arabidopsis, researchers have used RIL populations ranging from 30 to 420 lines averaging between 100 and 150 .

\section{FROM QTL TO GENE}

Cloning genes based on the map position of mutants (positional cloning) is very effective in Arabidopsis because of the efficiency with which a segregating population can be analyzed and the abundance of markers available [4]. Nowadays the mapping of a mutant down to less than $50 \mathrm{~kb}$ (on average 0.2 centi-Morgan) is sufficient to search for candidate genes. The ensuing DNA sequence comparison of the candidate genes between mutant and wild type will then reveal the precise location of the mutations. Complementation of the mutant phenotype after transformation of the mutant with the wild-type allele provides final proof that the gene causing the mutation has been correctly identified. Similar approaches have been used to clone QTLs. However, because positional cloning is done gene-by-gene, care has to be taken that only one segregating locus is studied at a time. This can be achieved by using so-called near isogenic lines (NILs), containing a relatively small introgression of one parent alleles at a QTL position into the genetic background of the other parent (recurrent parent). Subsequently, a population of lines with different recombinant events is selected by genotyping the offspring of a cross between the NIL and the recur- 
rent parent with polymorphic markers in the QTL region. The QTL is eventually fine-mapped by repeating this process with progeny lines that remain to show the phenotypic effect of the QTL. The procedure is then the same as when working with mutants as soon as the QTL has been fine-mapped to a sufficiently small region. Another approach is to make use of residual heterozygosity present at QTL in RILs, after several generations of selfing. The genetic background of the progeny of such lines is a mixture of both parental accessions. This so-called HIF (heterogeneous inbred families) concept [5] is effective because it does not require the isolation and identification of NILs and it is an efficient way when a phenotype depends on more than one locus from one of the parents (epistasis). By selecting lines that are homozygous for one of these regions but heterozygous for the other one still can study the genes individually. Using NILs and/or HIF will also allow the validation/confirmation of the presence and the effect of a QTL.

Accurate phenotyping QTLs with small effect and with larger environmental influence is difficult. Therefore, it is often more effective to select homozygous recombinants in a genomic region surrounding the QTL and then to analyse their phenotype in a large number of replicates. Such procedures are also described by Peleman et al. [6] and are commonly referred to as QTL Isogenic Recombinant Analysis.

A complication of QTL cloning compared to positional cloning of mutants is finding the gene(s) responsible for the phenotype among the candidate genes in the region where the QTL was fine-mapped. In case of mutants in a self-pollinating species such as Arabidopsis, any DNA sequence difference that is detected indicates that the gene has been identified. However, between Arabidopsis accessions, polymorphisms of sequence occur at an abundance of about one in every $350 \mathrm{bp}$ [7], even in coding regions. Thus, on average several differences will be found in each gene. Because of this, sequence information in most cases is not informative for the detection of the QTN (Quantitative Trait Nucleotide). However, sometimes the presence of deletions [8] or mutations in known important parts of the gene [9] are informative. Therefore, transformation experiments are crucial to ultimately proof which gene(s) is (are) responsible for the phenotypic differences observed between NILs or HIFs. The feasibility of this concept in Arabidopsis has been shown by the cloning of several QTLs affecting traits such as flowering time, frost tolerance, and seed dormancy (for review [2]).

Another resource to find candidate genes is the analysis of expression of genes in the QTL region. This can nowadays be done routinely using available microarrays or by conventional Northern blots or quantitative RT-PCR. When the functional allelic variation results in differences in gene expression, this may give a clear indication of the candidate gene, to be further proven with the approaches given above.

For the fine mapping of a QTL comprising the candidate genes, the following tools are currently available: expression analysis of the candidate genes, and the search for mutants that have mutations in these genes and exhibit the related phenotypes in the QTL region are the tools available at the moment. Because some tools are best developed for Arabidopsis and rice, QTL cloning is most efficient in these two species.

Based on a literature study of 20 QTLs cloned or accurately tagged in plants, it was concluded that mapping of quantitative traits is surprisingly accurate: the genes mapped within $2 \mathrm{cM}$ of the original QTL position [10].

\section{USE OF QTL ANALYSIS IN PHYSIOLOGICAL RESEARCH}

In this paragraph, a few examples of the application of QTL analysis in physiological research will be given, focussing on Arabidopsis as a model species.

The major application of QTL analysis is the localization and ultimately the identification of genes involved in complex quantitative traits. Arabidopsis is a long-day plant, flowering being promoted under long day (> 12-h day length). This trait is clearly a quantitative genetic trait: depending on accession and environmental conditions, the timing of flowering ranges from 3 weeks after planting up to more than 9 months. QTL analysis has revealed a large set of genes having major or minor effects on flowering time (reviewed in [1]). Some of the loci were known already from mutant analyses, but also new ones were revealed. Two of these genes, FRI and FLC, have been cloned and extensively studied but at the mechanistic and population genetics levels (reviewed in [11]).

The advantage of using immortal mapping populations in QTL analysis is that an unlimited number of traits can be studied with the same populations, possibly revealing co-location of QTL position for seemingly unrelated traits. Examples are the effect of $F L C$ on flowering time and circadian period length [12], and the effect of the ERECTA locus on plant length and water use efficiency by affecting stomatal densities [13] and the resistance to Plectosphaerella cucumerina [14]. Plant growth, being a clear example of a polygenic trait, has also been studied using QTL analysis, revealing colocations of sometimes unexpected traits [15-17]. Although such colocation might be caused by a common underlying gene, it may also be due to different, but closely linked genes. Therefore, fine mapping and cloning QTL is required to distinguish pleiotropic effects (a single gene affecting various traits) from the effects of linked genes, when QTL of several traits are co-localised. Furthermore, it is helpful in confirming or disproving unexpected co-location of QTL for diverse traits.

QTL mapping has been used to unravel genes involved in biochemical pathways. Evidently, when 


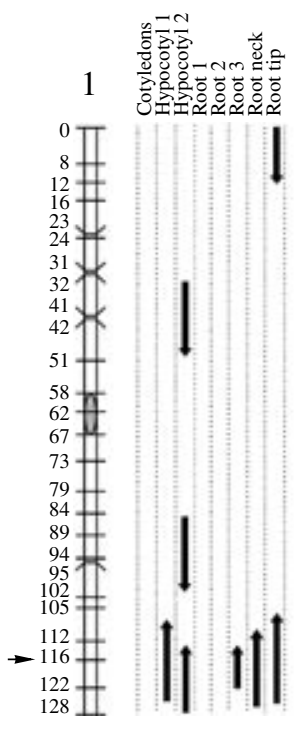

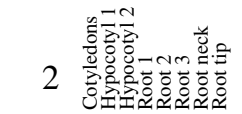
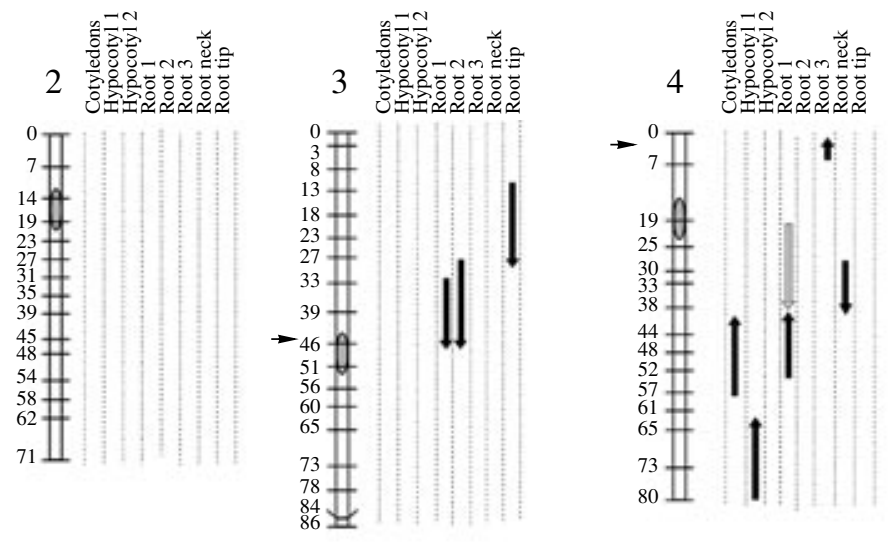

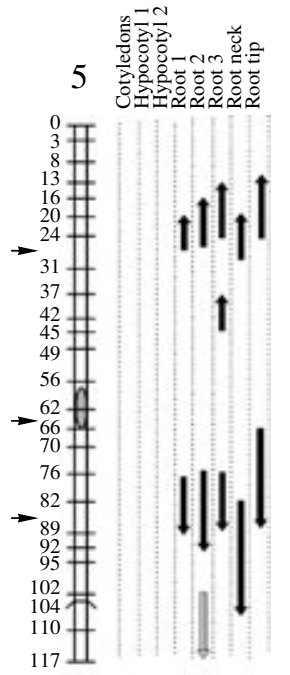

Fig. 2. Genetic map of Arabidopsis showing QTLs for activities of sucrose synthase, as calculated from histochemical assays, for various organs of seedlings.

Seven-day old seedlings were stained for Susy activity according to Sergeeva et al. [20]. Lengths of the arrows indicate the twoLOD intervals, and directions of the arrows indicate the allelic effects; upward: Ler alleles increase, downward: Cvi alleles increase trait values. Black arrows indicate QTL with significance $>95 \%$; gray arrows indicated QTL with significance $>90 \%$. Horizontal arrows indicate the approximate positions of annotated Susy genes.

allelic variation is present in genes encoding for biosynthetic enzymes, analyzing enzyme activity as a quantitative trait in a segregating population is expected to point towards the genes encoding the enzyme under investigation. Here, the advantage of using Arabidopsis is clear: the genome has been sequenced and many genes are (putatively) annotated. This has been shown, e.g. for enzymes involved in the biosynthesis of glucosinolates [18] and for phosphoglucomutase (PGM), converting glucose-1-phosphate into glucose-6-phosphate [19]. However, QTL analysis reveals more than structural and often already annotated genes: in the study on PGM activity, several loci were found that significantly affected the activity of the enzyme, whereas no PGM gene had been annotated in that chromosomal region. This suggests that the QTL points to regulators of the activity of the enzyme. The nature of such presumed regulators is not directly evident from the analysis: it might be transcription factors, allosteric affectors, or others. For instance, in the case of anthocyanin accumulation upon stress, the QTL was found to encode for a transcription factor regulating anthocyanin biosynthesis genes [9].

Figure 2 shows another example of QTL analysis of enzyme activity: the enzyme sucrose synthase (Susy), involved in the breakdown of sucrose by splitting it into UDP-glucose and fructose. Susy activities were determined in seedlings of Arabidopsis of the Landsberg erecta $\times$ Cape Verde Islands (Ler $\times$ Cvi) RIL population using a histochemical method [20]. These methods reveal (semi)quantitative data for tissue-specific activities, which can be used for QTL analysis [19, 21]. Several loci were observed that affect Susy activity, some of which were found in several, but not all, organs studied. For instance, at the lower end of chromosome 1 QTL for Susy activities were found in hypocotyl, root neck, and lower parts of the roots (root 3), but not in the upper regions of the roots (root 1 and root 2). Other QTL were confined to one or a few organs, e.g., at the top of chromosome 4, where a locus was found affecting activity in one region of the root only (Fig. 2).

In Arabidopsis six Susy genes have been annotated, partly based on sequence homologies [22]. All these genes are located within the two-LOD intervals of various QTLs, making them likely candidates for the observed variation. However, for various Susy QTLs, no Susy gene has been described in the 2-LOD interval, and hence the QTL is likely to be caused by another gene, the identity of which has to be revealed by further fine-mapping.

QTL analysis has also been used to link an overall phenotypic trait with the underpinning biochemical pathway, and the enzyme involved. Sergeeva et al. [21] determined lengths of roots and hypocotyls of seedlings of the Ler $\times$ Cvi RIL population [23]. For both traits, several QTLs were detected. Using the same population, the activities of soluble and cell-wall-bound invertase were determined. It appeared that a locus that had a major effect on the root length, also affected the soluble (vacuolar) invertase activity, especially in the lower parts of the roots. This locus did not affect hypocotyl length. Since the region contained an annotated invertase gene, this gene was an obvious candidate. Further fine-mapping and the use of a knock-out mutant for this gene confirmed its role in root elongation. 
The power of detecting QTLs for multiple traits has recently been demonstrated in a study combining largescale untargeted metabolomics and QTL analysis: using LC/MS, Keurentjes et al. [24] determined the levels of over 1000 metabolites in all lines of a RIL population. For over $75 \%$ of these metabolites one or more loci could be detected, significantly affecting their level. It was shown that correlations between QTLs for various metabolites could reveal relevant information on identity, and also on biochemical pathways. Similar "-omic" approaches are now be tested for genome-wide gene expression patterns and protein levels.

\section{TRANSLATION FROM MODEL TO CROP}

Based on the common molecular basis of many processes in plants, it is now becoming more obvious that many genes, for which the function is discovered in Arabidopsis, also underlie the genetic variation that is exploited by plant breeders in crop plants. However, in more distantly related species, similar processes may just as well be regulated by unrelated genes. The best known example is flowering time where QTL cloning in rice revealed a number of genes that previously had been shown to control flowering time in Arabidopsis, where this was based on mutant analysis [25]. However, the two major genes controlling flowering time variation in nature in Arabidopsis, which are FRI and $F L C$ are not found in monocots. In wheat, in which genes based on phenotype and physiology, were predicted to be similar to $F L C$, another regulatory gene was found [26]. For the major determinant of the day length response in barley, a gene was found that was not identified as a major player in Arabidopsis and rice [27], although the process in which the gene was involved (circadian rhythm) was known from Arabidopsis research. However, especially when dicot species are compared, many examples of similar genes controlling similar processes have been described.

Another possibility for exploitation of the vast amount research done on Arabidopsis and the availability of its genome sequence is to make use of synteny between genomes. With the complete genome sequence of rice now available, it has become clear that there are reasonably sized blocks of highly conserved synteny between this grass and Arabidopsis.

The level of synteny can be much higher between more related species. For instance, highly conserved genetic synteny has been reported up to the multi megabase scale between Arabidopsis and other members of the Brassicaceae $[28,29]$. The $F L C$ gene, a major regulator of flowering in Arabidopsis, has been found to be central in the vernalization response in Brassica napus $[30,31]$ demonstrating the feasibility of comparative genetics.

To conclude, QTL mapping is a powerful technique to unravel genes involved in complex polygenic traits.
The genetic resources available in Arabidopsis, and also in rice, allow rapid progress in these species, and translation of knowledge to other species has been shown to be feasible. QTL analysis may also be useful to provide markers for marker-assisted breeding, especially when the gene of interest is known: markers maybe developed in the gene of interest allowing accurate and rapid selection of superior genotypes.

\section{ACKNOWLEDGMENTS}

This work was supported by grants from the European Union (QLG2-CT-2001-01097), NATO (LG978850), Wageningen University, and NWO (B-86-192), and was partly carried out within the research program of the Centre for BioSystems Genomics (CBSG), which is a part of the Netherlands Genomics Initiative/Netherlands Organization for Scientific Research.

\section{REFERENCES}

1. Koornneef, M., Alonso-Blanco, C., and Vreugdenhil, D., Naturally Occurring Genetic Variation in Arabidopsis thaliana, Annu. Rev. Plant Biol., 2004, vol. 55, pp. 141172.

2. Alonso-Blanco, C., Mendez-Vigo, B., and Koornneef, M., From Phenotypic to Molecular Polymorphisms Involved in Naturally Occurring Variation of Plant Development, Int. J. Dev. Biol., 2005, vol. 49, pp. 717732.

3. Vreugdenhil, D., Aarts, M.G.M., and Koornneef, M., Exploring Natural Genetic Variation to Improve Plant Nutrient Content, Plant Nutritional Genomics, Broadley, M.R. and White, J. Eds., Oxford: Blackwell, 2005, pp. 201-219.

4. Lukowitz, W., Gillmor, C.S., and Scheible, W.-R., Positional Cloning in Arabidopsis. Why It Feels Good to Have a Genome Initiative Working for You? Plant Physiology, 2000, vol. 123, pp. 795-805.

5. Tuinstra, M.R., Ejeta, G., and Goldsbrough, P.B., Heterogeneous Inbred Family (HIF) Analysis, a Method for Developing Near-Isogenic Lines That Differ at Quantitative Trait Loci, Theor. Appl. Genet., 1997, vol. 95, pp. 1005-1011.

6. Peleman, J.D., Wye, C., Zethof, J., Sørensen, A.P., Verbakel, H., van Oeveren, J., Gerats, T., and van der Voort, J.R., Quantitative Trait Locus (QTL) Isogenic Recombinant Analysis, a Method for High-Resolution Mapping of QTL within a Single Population, Genetics, 2005, vol. 117, pp. 1341-1352.

7. Schmid, K.J., Sörensen, T.R., Stracke, R., Törjék, O., Altmann, T., Mitchell-Olds, T., and Weisshaar, B., Large-Scale Identification and Analysis of GenomeWide Single-Nucleotide Polymorphisms for Mapping in Arabidopsis thaliana, Genome Res., 2003, vol. 13, pp. 1250-1257.

8. Alonso-Blanco, C., Gomez-Mena, C., Llorente, F., Koornneef, M., Salinas, J., and Martinez-Zapater, J.M., Genetic and Molecular Analyses of Natural Variation Indicate $C B F 2$ as a Candidate Gene for Underlying a 
Freezing Tolerance Quantitative Trait Locus in Arabidopsis, Plant Physiol., 2005, vol. 139, pp. 1304-1312.

9. Teng, S., Keurentjes, J., Bentsink, L., Koornneef, M., and Smeekens, S., Sucrose-Specific Induction of Anthocyanin Biosynthesis in Arabidopsis Requires the MYB75/PAP1 Gene, Plant Physiol., 2005, vol. 139, pp. 1840-1852.

10. Price, A.H., Believe It or Not, QTLs Are Accurate, Trends Plant Sci., 2006, vol. 11, pp. 213-216.

11. Sung, S. and Amasino, R.M., Remembering Winter: Towards a Molecular Understanding of Vernalization, Annu. Rev. Plant Biol., 2005, vol. 56, pp. 491-508.

12. Swarup, K., Alonso-Blanco, C., Lynn, J.R., Michael, S.D., Amasimo, R.M., Koornneef, M., and Millar, A.J., Natural Allelic Variation Identifies New Genes in the Arabidopsis Circadian System, Plant J., 1999, vol. 20, pp. 6777.

13. Masle, J., Gilmore, S.R., and Farquhar, G.D., The ERECTA Gene Regulates Plant Transpiration Efficiency in Arabidopsis, Nature, 2005, vol. 11, pp. 866-870.

14. Llorente, F., Alonso-Blanco, C., Sánchez-Rodriguez, C., Jorda, L., and Molina, A., ERECTA Receptor-Like Kinase and Heterotrimeric G Protein from Arabidopsis Are Required for Resistance to the Necrophic Fungus Plectosphaerella cucumerina, Plant J., 2005, vol. 43, pp. 165-180.

15. El-Lithy, M.E., Clerkx, E.J., Ruys, G.J., Koornneef, M., and Vreugdenhil, D., Quantitative Trait Locus Analysis of Growth-Related Traits in a New Arabidopsis Recombinant Inbred Population, Plant Physiol., 2004, vol. 135, pp. 444-458.

16. Loudet, O., Chaillou, S., Merigout, P., Talbotec, J., and Daniel-Vedele, F., Quantitative Trait Loci Analysis of Nitrogen Use Efficiency in Arabidopsis, Plant Physiol., 2003, vol. 131, pp. 345-358.

17. Loudet, O., Chaillou, S., Krapp, A., and Daniel-Vedele, F., Quantitative Trait Loci Analysis of Water and Anion Contents in Interaction with Nitrogen Availability in Arabidopsis thaliana, Genetics, 2003, vol. 163, pp. 711722.

18. Kliebenstein, D.J., Kroymann, J., and Mitchell-Olds, T., The Glucosinolate-Mysosinase System in an Ecological and Evolutionary Context, Curr. Opi. Plant Biol., 2005, vol. 8, pp. 264-271.

19. Sergeeva, L.I., Vonk, J., Keurentjes, J.J.B., van der Plas, L.H.W., Koornneef, M., and Vreugdenhil, D., Histochemical Analysis Reveals Organ-Specific Quantitative Trait Loci for Enzyme Activities in Arabidopsis, Plant Physiol., 2004, vol. 134, pp. 237-245.

20. Sergeeva, L.I. and Vreugdenhil, D., In Situ Staining of Activities of Enzymes Involved in Carbohydrate Metabolism in Plant Tissues, J. Exp. Bot., 2002, vol. 53, pp. 361-370.

21. Sergeeva, L.I., Keurentjes, J.J.B., Bentsink, L., Vonk, J., van der Plas, L.H.W., Koornneef, M., and Vreugdenhil, D.,
Vacuolar Invertase Regulates Elongation of Arabidopsis thaliana Roots as Revealed by QTL and Mutant Analysis, Proc. Natl. Acad. Sci. USA., 2006, vol. 103, pp. 2994-2999.

22. Baud, S., Vaultier, M.-N., and Rochat, C., Structure and Expression Profile of the Sucrose Synthase Multigene Family in Arabidopsis, J. Exp. Bot., 2004, vol. 55, pp. 397-409.

23. Alonso-Blanco, C., Peeters, A.J.M., Koornneef, M., Lister, C., Dean, C., van den Bosch, N., Pot, J., and Kuiper, M.T.R., Development of an AFLP Based Linkage Map of Ler, Col and Cvi Arabidopsis thaliana Ecotypes and Construction of a Ler/Cvi Recombinant Inbred Line Population, Plant J., 1998, vol. 14, pp. 259271.

24. Keurentjes, J.J.B., Fu, J., de Vos, C.H.R., Lommen, A., Hall, R.D., Bino, R.J., van der Plas, L.H.W., Jansen, R.C., Vreugdenhil, D., and Koornneef, M., The Genetics of Plant Metabolism, Nature Genet., 2006, vol. 38, pp. 842-849.

25. Hayama, R. and Coupland, G., The Molecular Basis of Diversity in the Photoperiodic Flowering Responses of Arabidopsis and Rice, Plant Physiol., 2004, vol. 135, pp. 677-684.

26. Yan, L., Loukoianov, A., Blechl, A., Tranquilli, G., Ramakrishna, W., San Miguel, P., Bennetzen, J.L., Echenique, V., and Dubcovsky, J., The Wheat VRN2 Gene Is a Flowering Repressor Down-Regulated by Vernalization, Science, 2004, vol. 303, pp. 1640-1644.

27. Turner, A., Beales, J., Faure, S., Dunford, R.P., and Laurie, D.A., The Pseudo-Response Regulator Ppd-H1 Provides Adaptation to Photoperiod in Barley, Science, 2005, vol. 310, pp. 1031-1033.

28. Lukens, L., Zou, F., Lydiate, D., Parkin, I., and Osborn, T., Comparison of a Brassica oleracea Genetic Map with the Genome of Arabidopsis thaliana, Genetics, 2003, vol. 164, pp. 359-372.

29. Parkin, I.A.P., Gulden, S.M., Sharpe, A.G., Lukens, L., Trick, M., Osborn, T.C., and Lydiate, D.J., Segmental Structure of the Brassica napus Genome Based on Comparative Analysis with Arabidopsis thaliana, Genetics, 2005, vol. 171, pp. 765-781.

30. Osborn, T.C., Kole, C., Parkin, I.A.P., Sharpe, A.G. and Kuiper, M., Lydiate, D.J., Trick, M., Comparison of Flowering Time Genes in Brassica rapa, B. napus and Arabidopsis thaliana, Genetics, 1997, vol. 146, pp. 11231129.

31. Tadege, M., Sheldon, C.C., Helliwell, C.A., Stoutjesdijk, P., Dennis, E.S. and Peacock, W.J., Control of Flowering Time by FLC Orthologues in Brassica napus, Plant J., 2001, vol. 28, pp. 545-553. 